\title{
Using a Non-Conforming Meshes Method to Simulate an Interaction Between Incompressible Flow and Rigid and Elastic Boundaries
}

\author{
Anas ABID MATTIE*, As'ad ALIZADEH** \\ *Department of Mechanics, Duhok Technical Institute, Duhok Polytechnic University, Kurdistan Region, Iraq, \\ E-mail: anas.mattie@dpu.edu.krd \\ **Department of Mechanical Engineering, College of Engineering, University of Zakho, Zakho, Iraq, \\ E-mail: alizadeh.a.info@gmail.com (Corresponding Author)
}

cross $^{\text {ref }}$ http://dx.doi.org/10.5755/j01.mech.25.4.22975

\section{Introduction}

Whenever a fluid field with a common boundary with a rigid or flexible solid is influenced by the fluid regime and the dynamic behavior of the solid field, the phenomenon of fluid-solid interaction has occurred. In recent years, studies on fluid and moving solid interactions have attracted many attentions. From gridding point of view, there are two types of conforming and non-conforming meshes. In the conforming meshes approach, fluid calculations take place on a boundary-oriented grid. It is therefore quite obvious that the creation and production of a suitable conforming meshes is very importance. An important advantage of this method is to applying of boundary conditions is the best. The major disadvantage of this gridding method is to maintain the accuracy and stability of the calculation. This subject was so important that so far researches and scientists have done the best on gridding which have been the subject of many books and articles [1]. In a non-conforming meshes method, there is no need of complex gridding matching with boundary. Therefore, a streaming flow analysis can be done with a simple grid. Many methods have been proposed based on this model, the most important of which are: Cartesian grid method, immersed interface method and immersed boundary method [2-4]. Unlike the immersed boundary method with numerical smearing near the interface, the immersed interface method (IIM) can capture the solution and its derivative jumps sharply and maintains second-order accuracy via incorporating the known jump conditions into the finite difference approximations near the interface. The advantage of this method over other computational fluid dynamics methods is that there is no compulsion to adapt the computational grid with the immersed boundaries. So, the flow field can be analyzed numerically using a simple Cartesian mesh domain. In general, the grid points aren't coincided to the immersed intersection. The main idea of this method (Immersed Interface Method) is solving the Navier-Stokes equations over the uniform Cartesian grids. The IIM is introduced by Le Veque and $\mathrm{Li}$ [5] to solve the elliptic equations. Then, this method was developed for Stokes flows with the elastic boundaries or surface tension [6]. This method (IIM) was extended to deal with the flexible border problems using the Navier-Stokes equations [7]. The immersed interface method was developed further for the Navier-Stokes equations in [8,9]. The IIM was also used in $[10,11]$ for solving the two-dimensional streamfunctionvorticity equations on irregular domains. Xu and Wang [12] have extended the IIM to the 3D Navier-Stokes equation for simulating fluid-solid interaction. Cartesian grid approach has been presented by Ye et al. [13] and Udaykumar et al. [14] using finite volume techniques. They reshaped the immersed boundary cells and use a polynomial interpolating function to approximate the fluxes and gradients on the faces of the boundary cells while preserving second-order accuracy. Many of the natural, engineering, and medical phenomena include issues of fluid-structure interaction. In recent years, various numerical methods have been used for this purpose. Jayathilake et al. [15] Used the immersed interface method to study two-dimensional thin-walled capsule of a flexible membrane is adhered onto a rigid planar substrate under adhesive forces (derived from a potential function) in the presence of osmosis across the membrane. Gurugubelli et al. [16] developed a new high-order finite element Coupled Field with Explicit Interface (CFEI) code has been developed for simulating flapping motion of a thin flexible body in a uniform flow with strong add-mass effects. Li et al. [17] simulated the two-dimensional flows past a stationary circular cylinder near a plane boundary are numerically simulated using an immersed interface method with second-order accuracy. Bagchi [18] simulated the flow of blood in small vessels based on the immersed boundary method. In his work, red blood cells are considered as liquid capsules. Also they [19] examined the deformation effects of several red blood cells in the shear flow. Kaoui et al. [20] investigated the effects of viscosity inside and outside the viscole on membrane deformation in simple microchannel and eclipse using Boltzmann's method. With the help of this method, Ghafuri et al. [21] simulated the movement of a red blood cell in a barrier microchannel. In their work, the change in membrane hardness is considered to be an important factor in changing hematocrit of the blood. They [22] also examined a circular capsule motion in the shear and poiseuille flow and examined the effect of the capsule hardness on the flow velocity profile. In this study, using immersed interface method, which is a non-conforming meshes method. we study the behavior of the flow around the rigid and elastic boundaries and compare the ability of this method to simulate the fluid-solid interaction with other experimental and numerical results. The purpose of this study is the ability of the immersed interface method to be used as a non-conforming meshes method to simulate the flow around rigid and flexible borders (such as red blood cells).

\section{Governing equations}

$£$ is a two-dimensional area of the fluid field, which includes the material interface. Navier-Stokes equations for the incompressible flow are as follows: 


$$
\begin{aligned}
& \nabla \cdot \vec{u}=0 \\
& \rho\left(\frac{\partial \vec{u}}{\partial t}+\vec{u} \cdot \nabla \vec{u}\right)=-\nabla p+\eta \nabla^{2} \vec{u}+\vec{F} .
\end{aligned}
$$

with boundary and initial condition [23]:

$$
\begin{aligned}
& \left.u\right|_{\partial \mathrm{E}}=u_{\text {body }} \\
& u(x, 0)=u_{\text {body }}
\end{aligned},
$$

where: $u$ is the fluid velocity, $p$ is the pressure, $\rho$ is the density, and $\mu$ the viscosity of the fluid.

The force $F$ which has the form:

$$
\vec{F}(\vec{x}, t)=\int_{r(t)} f(s, t) \delta(\vec{x}-\vec{X}(s, t)) d s,
$$

where: $\vec{X}(s, t)$ is the arc-length parameterization of $\Gamma(t)$ is the arc-length, $x(x, y)$ is spatial position, and $f(s, t)$ is the force density. Here, $\delta(x)$ is the Dirac function. mulation:

The motion of the interfaces is shown as below for-

$$
\vec{u}(\vec{X}(s, t), t)=\frac{\partial \vec{X}(s, t)}{\partial t}=\int_{\mathrm{E}} \vec{u}(\vec{x}, t) \delta(\vec{x}-\vec{X}(s, t)) d \vec{x}
$$

The force density using an expression of the form:

$$
\vec{f}(s, t)=k\left(\vec{X}^{e}(s)-\vec{X}(s, t)\right)
$$

where: $k$ is a constant, $k \gg 1$, and $\vec{X}^{e}$ is the arc-length parameterization of the required boundary position. The forcing term in Eq. (6) is a particular case of the feedback forcing formulation proposed by Goldstein et al. [24] with $\beta=0$. In [21], the force is expressed as:

$$
\vec{f}(s, t)=\alpha \int_{0}^{t} \vec{U}\left(s, t^{\prime}\right) d t^{\prime}+\beta \vec{U}(s, t),
$$

where: $\vec{U}$ is the velocity at the control points; $\alpha$ and $\beta$ are chosen to be negative and large enough so that $\vec{U}$ will stay close to zero.

The Navier-Stokes equations are discretized using a standard finite difference method on a staggered Cartesian grid. Cartesian grid is used for solving interface problems or problems with sophisticated geometry have become popular recently. Existing Cartesian grid methods for interface problems can be classified into two general groups: methods that determine the jump conditions across the interface and include them into the finite difference scheme and our method which is based on the immersed interface method originally proposed by LeVeque and $\mathrm{Li}[5,25]$ falls into the first group. Other methods smooth out the singular force before it is applied to the fluid. When singular forces are applied on a material interface, the solutions of the Navier-Stokes equations may be non-smooth or discontinuous across the interface. Let $n$ and $\tau$ be the unit outward normal and tangential vectors to the interface, respectively. The respective normal and tangential components of the force density $f_{1}=\vec{f}(s, t) \cdot \vec{n}$ and $f_{2}=\vec{f}(s, t) \cdot \vec{\tau}$ can be related to the jump conditions for pressure and velocity as follows [8]:

$$
[\vec{u}]=0,\left[\mu \vec{u}_{\xi}\right]=-f_{2} \vec{\tau},\left[\vec{u}_{\tau}\right]=0
$$

$$
\begin{aligned}
& {[p]=f_{1},\left[p_{\xi}\right]=\frac{\partial f_{2}}{\partial s},\left[p_{\eta}\right]=\frac{\partial f_{1}}{\partial s},} \\
& {\left[\mu \vec{u}_{\xi \xi}\right]=k f_{2} \vec{\tau},\left[\mu \vec{u}_{\xi \eta}\right]=-\frac{\partial f_{2}}{\partial \eta} \vec{\tau}-k f_{2} \vec{n},} \\
& {\left[\mu \vec{u}_{\xi \xi}\right]=-\left[\mu \vec{u}_{\eta \eta}\right]+\left[p_{\eta}\right] \vec{\tau}-\rho\left[\overrightarrow{u_{\xi}}\right] \vec{u} \cdot \vec{n} .}
\end{aligned}
$$

The above equations were derived in [8] and here, we have used the same notation for clarity. The jump, denotes the difference between the value of its argument outside and inside the interface, and $(\xi, \eta)$ are the rectangular coordinates associated with the directions of $\vec{n}$ and $\vec{\tau}$, respectively. Also, $k$ is the signed valued of the curvature of the interface. We assume that $\vec{n} \times \vec{\tau}=\vec{k}=$ constant, so that $\vec{n}$ can point either towards, or outwards from, the center of curvature. We note that from above terms the values of the jumps of the first and second derivatives of velocity and pressure taken with respect to the $(x, y)$ coordinates are achieved by a simple coordinate transformation. We consider the below relations as a simple:

$$
\begin{aligned}
& {\left[\vec{u}_{x}\right]=\left[\vec{u}_{\xi}\right] n_{1}+\left[\vec{u}_{\xi}\right] \tau_{1},} \\
& {\left[\vec{u}_{y y}\right]=\left[\vec{u}_{\xi \xi}\right] n_{2}^{2}+2\left[\vec{u}_{\xi \eta}\right] n_{2} \tau_{2}+\left[\vec{u}_{\xi \eta}\right] \tau_{2}^{2},}
\end{aligned}
$$

where: $\vec{n}=\left(n_{1}, n_{2}\right)$ and $\vec{\tau}=\left(\tau_{1}, \tau_{2}\right)$ are the Cartesian components of the normal and tangential vectors to the interface at the point considered.

\section{Results and discussion}

3.1. The effect of increasing Reynolds number on aerodynamic forces on rigid boundaries and flow parameters

In this section, we investigate the interaction of the flow and a circular ridge boundary located at the center of the channel. The flow characteristics depend on the Reynolds number, in which $R e=D u_{\infty} / v \quad u_{\infty}$ is the inlet velocity, $D$ is the cylinder diameter and $v$ is the kinematic viscosity of the fluid. The flow in Reynolds numbers of 30 and 40 is steady state and has a reversed flow in the wake region. The wake dimensions are determined by the length of wake $(l)$, the distance from the center of the vortex to the closest point in the cylinder surface in the direction of flow $(a)$, the transverse distance between the two vertex centers $(b)$, and the angle of the flow separation (in degrees) $\theta$, as shown in Fig. 1.

The stream lines, vorticity contour are shown in Figs. 2 and 3. Table 1 shows the wake dimensions and the drag coefficients and the results have been compared with 
other numerical and experimental results that are in good agreement. By increasing Reynolds number, $l, a$ and $b$ increase. It is clear from the following figures that when the Reynolds number increases, the size of the vortices become larger and the flow separation angle $\theta$ is also increased. Also, when the Reynolds number increases, the drag coefficient decreases. In the case of small Reynolds numbers $R e<5$, the flow around the cylinder stocks to it completely and the separation phenomenon does not occur. By increase of the Reynolds Number, the flow separates from cylinder edges and two symmetrical vortices behind the cylinder is generated. These two vortices are of the same magnitude and the opposite direction of rotational motion. By increasing the Reynolds number, the symmetrical vortices behind the cylinder become oscillating and non-stable, which is the phenomenon of vortex shedding (Von Carmen Street).

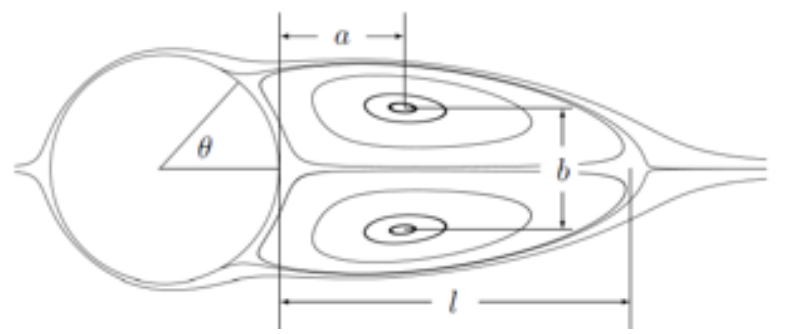

Fig. 1 Introduction of problem variables

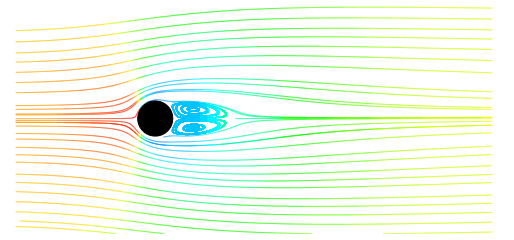

a

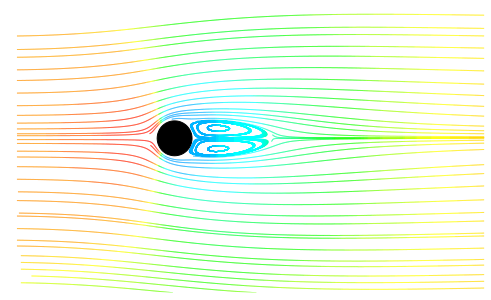

b

Fig. 2 Stream lines for (a) $R e=30$ and (b) $R e=40$

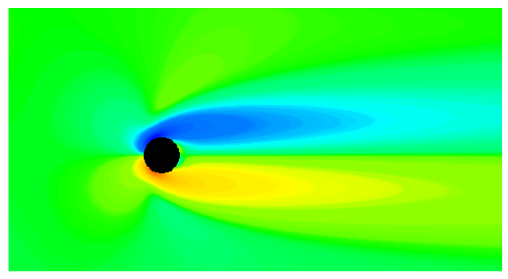

a

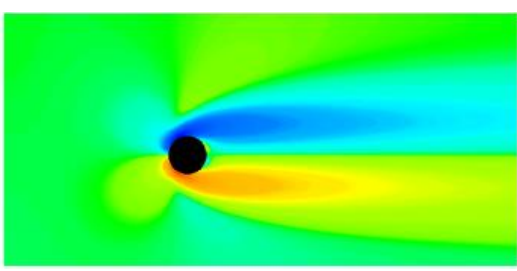

b

Fig. 3 Vorticity Contours for (a) $R e=30$ and (b) $R e=40$
The coefficient of pressure $C_{p}$ along the cylinder surface at $R e=40$ is shown in Fig. 4 , where $\theta=0^{\circ}$ corresponds to the stagnation point. Comparing the results with the Braza results [29] shows good agreement. The wall coefficient of pressure is defined as: $C_{p}=\frac{p-p_{\infty}}{\frac{1}{2} p u_{\infty}^{2}}$ where $P_{\infty}$ is the far flow pressure and $p$ is the pressure on the cylinder surface. From the stagnation point to the $90^{\circ}$ angle, as the stream lines become closer, the flow velocity increases as a result $u=\frac{\partial \psi}{\partial y}$ and the pressure or $C_{p}$ is decreased. At an angle of $90^{\circ}$ to $180^{\circ}$, due to the fact that the distance between the lines becomes more, it results in slow down and increase in $C_{p}$. The minimum value of the $C_{p}$ is $90^{\circ}$.

Table 1

Comparison of the present results with other numerical and experimental results

\begin{tabular}{|c|c|c|c|c|c|c|}
\cline { 2 - 7 } \multicolumn{1}{c|}{} & $\begin{array}{c}\text { Experimental } \\
\text { and numerical } \\
\text { results }\end{array}$ & $1 / D$ & $a / D$ & $b / D$ & $\theta$ & $C_{D}$ \\
\hline \multirow{2}{*}{$R e=30$} & $\begin{array}{c}\text { Countanceau et } \\
\text { al. [26] }\end{array}$ & 1.55 & 0.54 & 0.54 & 50 & - \\
\cline { 2 - 8 } & Pinelli et al. [27] & 1.70 & 0.56 & 0.52 & 48.1 & 1.80 \\
\cline { 2 - 8 }$R e=40$ & Present study & 1.67 & 0.58 & 0.52 & 45 & 1.78 \\
\hline & $\begin{array}{c}\text { Countanceau et } \\
\text { al. [26] }\end{array}$ & 2.13 & 0.76 & 0.59 & 53.8 & - \\
\cline { 2 - 8 } & Wang et al. [28] & 2.36 & 0.72 & 0.6 & 53.8 & 1.54 \\
\cline { 2 - 8 } & Present study & 2.38 & 0.77 & 0.59 & 52 & 1.58 \\
\hline
\end{tabular}

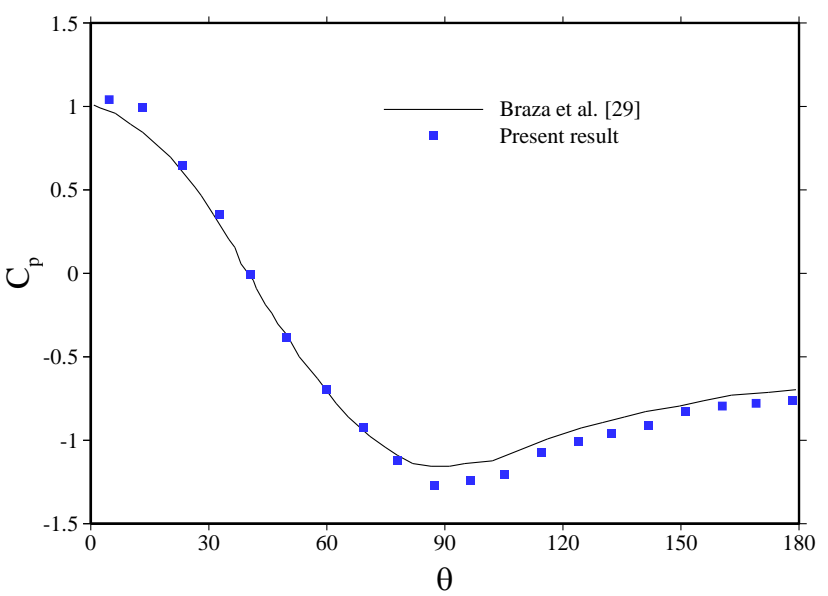

Fig. 4 Coefficient of pressure along the cylinder surface at $\operatorname{Re}=40$

By increasing the Reynolds number to $R e=100$ and 200, the flow is unstable and the periodic loss of the vortex is observed. The well-known Von Karman street is shown in Fig. 6. Figures 5 and 6 show the stream lines, vorticity contour respectively. Vortex shedding frequency is $f_{s}$. The Strouhal number $S t=\frac{D f_{s}}{u_{\infty}}$ and the average drag coefficient are compared with other numerical results in Table 2, which is in good agreement. It can be seen that with the increase in Reynolds number, the average drag coefficient decreases, but the Strouhal number increases. In fact, Strouhal 
number indicates the non-dimensional frequency of the alternating vortices shedding. Increasing the Reynolds number increases the rate of the vortices spreading. That is, in a certain time, more vortices are spread in the flow.

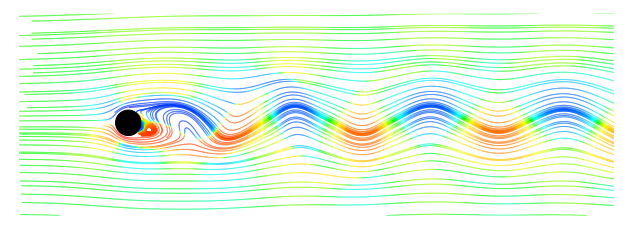

a

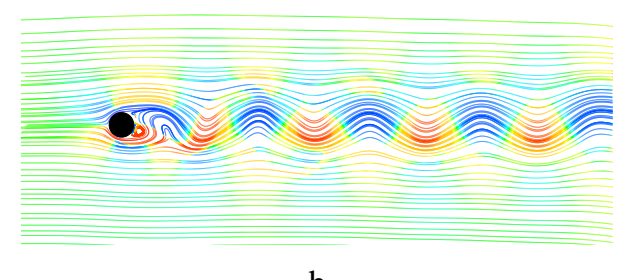

b

Fig. 5 Stream lines for (a) $R e=100$ and (b) $R e=200$

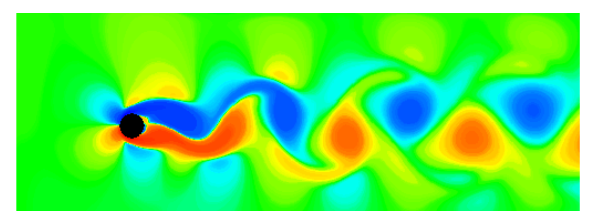

a

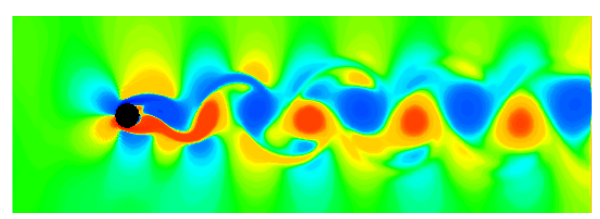

b

Fig. 6 Stream lines for (a) $\operatorname{Re}=100$ and (b) $R e=200$

The coefficient of pressure $C_{P}$ at $R e=100$ is shown in Fig. 7. Comparing the results with the Braza results [29] shows good agreement. In this case, due to the fact that the vortex shedding (Karmen Street) has occurred, the stream lines are less inferior to the previous one, resulting in a lesser angle with a lower velocity and a higher pressure on the cylindrical surface. Therefore, the $C_{p}$ at a smaller angle (about $80^{\circ}$ ) will have a minimum value compared to the previous one. In the previous case $(R e=40)$, this value was obtained at $90^{\circ}$.

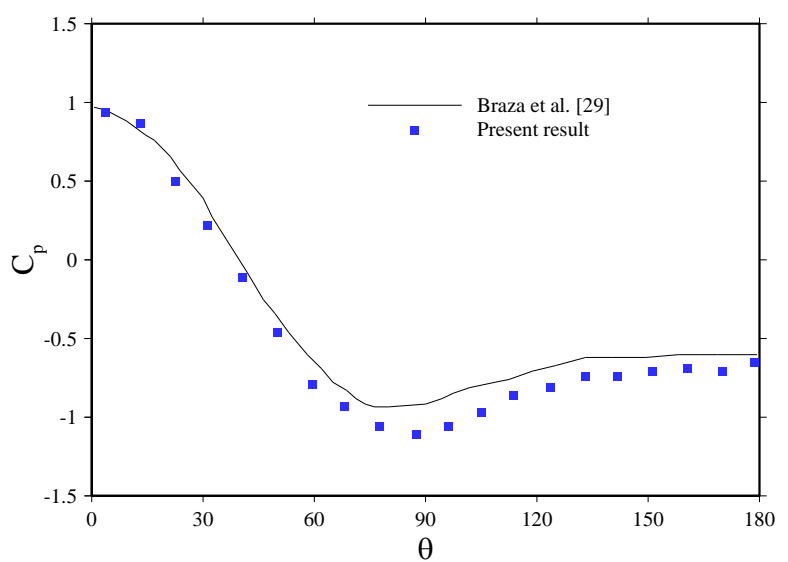

Fig. 7 Coefficient of pressure along the cylinder surface at $R e=100$
Table 2

Comparison of the present results with other numerical and experimental results

\begin{tabular}{|c|c|c|c|}
\cline { 3 - 4 } \multicolumn{2}{c|}{} & $\bar{C}_{D}$ & $S t$ \\
\hline \multirow{3}{*}{$R e=100$} & Braza et al. [29] & 1.359 & 0.16 \\
\cline { 2 - 4 } & Ji et al. [30] & 1.376 & 0.169 \\
\cline { 2 - 4 } & Present result & 1.380 & 0.16 \\
\hline \multirow{3}{*}{$R e=200$} & Braza et al. [29] & 1.386 & 0.20 \\
\cline { 2 - 4 } & Ji et al. [30] & 1.354 & 0.20 \\
\cline { 2 - 4 } & Present result & 1.355 & 0.20 \\
\hline
\end{tabular}

3.2. Behavior of circular elastic boundary in shear flow

In this section, the circular elastic boundary has been studied in shear flow. $E_{B}$ is a dimensionless bending modulus. The Taylor deformation parameter is defined as $D_{x y}=\frac{L-B}{L+B}$. In Fig. 8 a, the $L, B$ and $\theta$ parameters are shown. By increasing the bending modulus (increasing the hardness of the membrane), the Taylor deformation parameter decreases (Fig. 8 b), which indicates a lower membrane deformation. Also, as shown in Fig. $8 \mathrm{c}$, with decrease in the bending modulus, the angle $\theta / \pi$ is diminished.

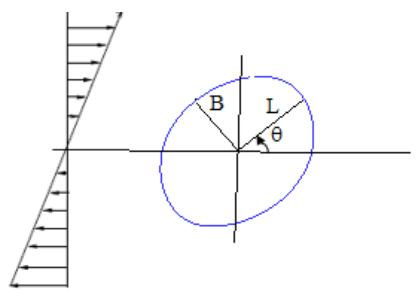

a

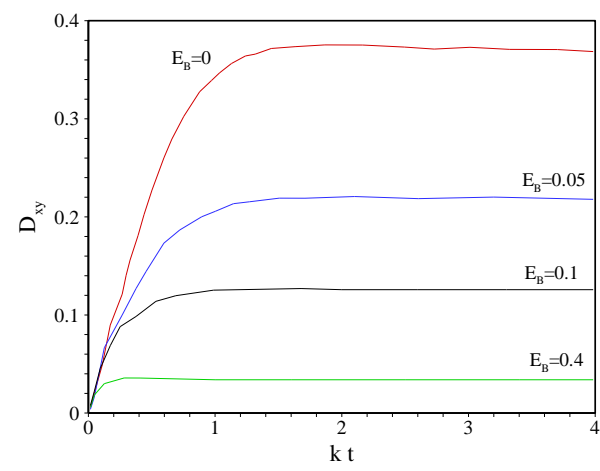

b

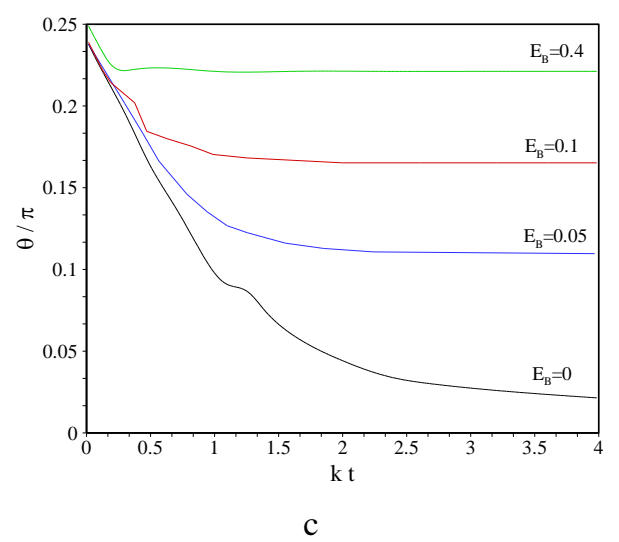

Fig. $8 \mathrm{a}$ - Introduction of the problem parameters; $\mathrm{b}-$ the Taylor deformation parameter; $\mathrm{c}-$ the angle $\theta / \pi$ 
In Fig. 9, the stream lines of flow related to the membrane with different bending modulus are observed. As shown, due to the fact that the direction of motion of the upper and lower walls are opposite, two vortices are created around the flexible membrane. In fact, these are vortices that rotate the membrane around the center called the TankTreading motion which is also observed in experimental observations [31]. The more flexible the membrane (the reduction of the bending modulus), the two vortices around it combine to form a larger vortex. In fact, it can be said that as hard as the membrane is, the immersed body prevents the communication of two smaller vortices. So it acts as a harder barrier, while for a more flexible membrane (Fig. 9 d), a larger vortex is formed around the body.

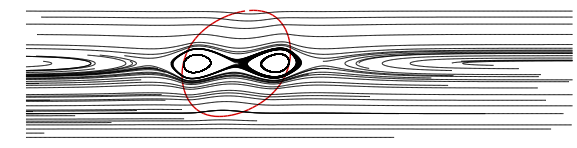

a

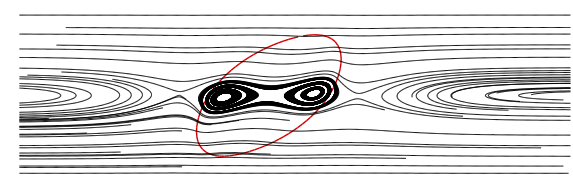

b

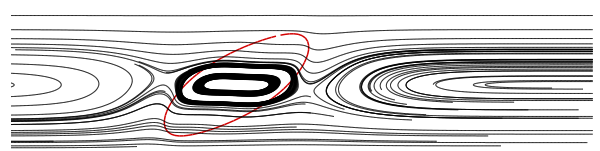

c

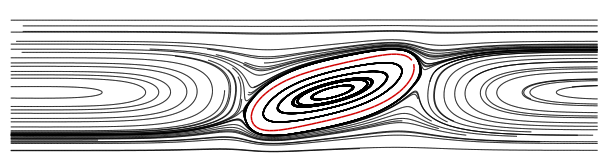

d

Fig. 9 Stream lines for Membrane with $E_{B}=0.4-$ a; $E_{B}=0.1-\mathrm{b} ; E_{B}=0.05-\mathrm{c} ; E_{B}=0.0-\mathrm{d}$

3.3. The dynamic behavior of an elastic membrane (red blood cell) inside a microvessel

In this section, we study the dynamical behavior of an elastic membrane (red blood cell) in a stenosed capillary. The purpose of this section is to investigate the effect of the membrane (cell) hardness on its deformation. In Figure10, one can see that the sick cell (with a tensile and bending modulus $6 \times 10^{-5} \mathrm{~N} / \mathrm{m}$ and $4 \times 10^{-18} \mathrm{~N} . \mathrm{m}$, respectively), makes smaller deformation, while the normal cell (with a tensile and bending modulus $6 \times 10^{-6} \mathrm{~N} / \mathrm{m}$ and $2 \times 10^{-19} \mathrm{~N}$.m, respectively), is more deformed and easier passes the stenosis. Normal cell moves faster than the sickle cell [32]. The sick cell, due to the harder membrane, blocks the fluid flow more easily than the normal cell through the stenosed part, and this results in reduction of the flow rate in stenosis. This behavior is caused by a type of disease called sickle cell anemia, in which cells become sickened and more rigid and, due to their distorted form, they lose oxygen delivery ability [33]. When the cell is sick, flow rate $\frac{Q}{Q_{0}}$ in the middle section of the channel is equal to 0.19 , while this number for the normal cell equlas to $0.31 . Q_{0}$ is as flow rate microvessel without cell (Fig. $10 \mathrm{c}$ ). Fig. $10 \mathrm{c}$ shows that the normal cell more elongated in the direction of flow and width of less than sick cell. Anemia is the most common symptom of sickle cell disease. In this disease, RBCs are produced in the form of sickle cells, but they lose the ability to carry oxygen as a result of their deterioration. As a result, the body lost water and suffered fever. The sickle cell causes hardening of the cells and trapping them in the vessels. As a result, the cells are degraded in spleen or abnormal due to onusual function, and the decrease in RBCs results in anemia. Anemia makes the body tired and pale, and the ability to carry oxygen to tissues will be difficult as a result. In many pathological conditions such as heart disease, anemia, malaria and flexibility of red blood cells reduced. changes in the mechanical properties of red blood cells with disorders happened in the microscale system, such as capillaries, and it disorder the functioning of vital organs such as the brain and the kidneys. The determination of hemodynamic changes is a major step in the development of new methods for the diagnosis and treatment of these diseases. Understanding the biological system and examining various parameters affecting the flow of fluid caused by the immersed boundary can lead to the design of medical devices.

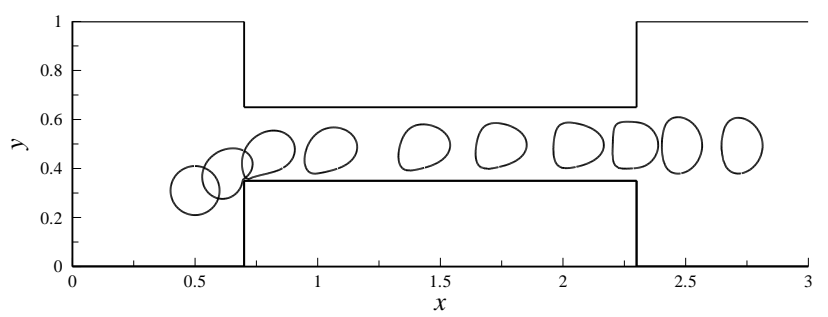

a

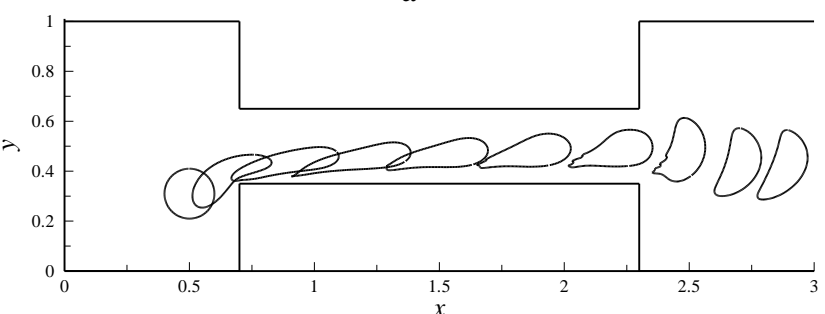

b

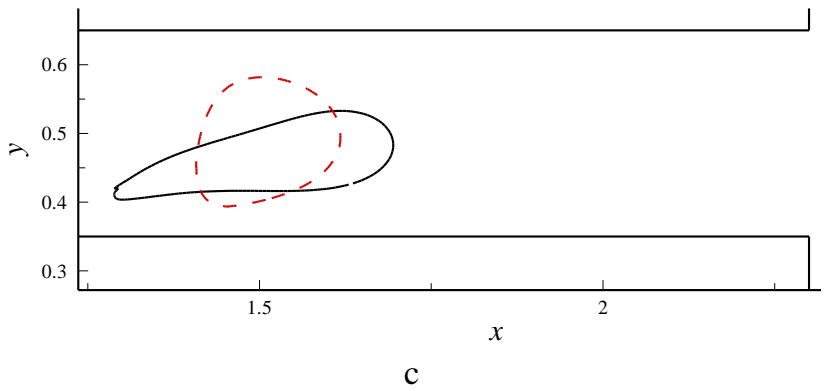

Fig. 10 Deformation sick cell - a; deformation normal cell - b; compare deformation sick and normal cells - c

\section{Conclusions}

In this study, used immersed interface method, which is a non-conforming meshes method.The immersed interface method is employed to solve the Navier-Stokes equations by using the finite different method on a staggered Cartesian grid. The basic idea of the immersed interface 
method is to account for the singular forces along the immersed boundaries by explicitly incorporating the jumps in the solutions and their derivatives into the difference equations. By doing that the IIM can avoid smearing sharp interfaces and maintains second order accuracy. First, the flow around a circular cylinder was simulated. As the Reynolds number rises, the vortex dimensions become larger and, as a result, the separation angle of the flow increases. Also, with the Reynolds number increasing, the drag coefficient decreases and the Strouhal number increases and the flow separated from cylinder and two symmetrical vortices is generated behind the cylinder. These vortices are of the same sizes and the direction of rotational motion is the opposite. By increasing the Reynolds number, the symmetrical vortices behind the cylinder are pulled out and become oscillating, which is the phenomenon of vortex shedding. The behavior of an elastic boundary in shear flow was investigated. It was observed that by increasing bending modulus (increasing stiffness) of body the shape change of the boundary decreases. As well as the tank-treading motion is also observed that this type of movement has been confirmed in experiments. Also observed that the sick cell makes smaller deformation, while the normal cell is more deformed and easier passes the stenosis. This results in reduction of the flow rate in stenosis. This behavior is caused by a type of disease called sickle cell anemia. Innovation of present work is investigating of the hardness of the red blood cell membrane on its deformation and the flow rate of a microchannel with eclipse using the Immersed Interface Method. Using immersed interface method, to observe the motion of the tank treading of an elastic membrane in linear shear flow is another innovation of this research, as seen in other laboratory and numerical results.

\section{References}

1. Versteeg, H. K.; Malalasekera, W. 2007. An Introduction to Computational Fluid Dynamics, Second Edition, London.

2. Anderson, D. M.; McFadden, G. B.; Wheeler, A. A. 1998. Diffuse-interface methods in fluid mechanics, Annual Review of Fluid Mechanics 30:139-165. https://doi.org/10.1146/annurev.fluid.30.1.139.

3. Angot, P. C.; Frabrie, P. 1999. Apenalization method to take into account obstacles in viscous flows, Numerische Mathematik 81:497-520. https://doi.org/10.1007/s002110050401.

4. Balaras, E. 2004. Modeling complex boundaries using an external force field on fixed Cartesian grids in largeeddy simulations, Computers \& Fluids 33:375-404. https://doi.org/10.1016/S0045-7930(03)00058-6.

5. LeVeque, R. J.; Li, Z. 1994. The immersed interface method for elliptic equations with discontinuous coefficients and singular sources, SIAM Journal on Numerical Analysis 31:1019-1044 https://doi.org/10.1137/0731054.

6. Lee, L. 2003. An immersed interface method for incompressible Navier-Stokes equations, SIAM Journal on Scientific Computing 25:832-856. https://doi.org/10.1137/S1064827502414060.

7. Li, Z.; Lai, M. C. 2001. The immersed interface method for the Navier-Stokes equations with singular forces, Journal of Computational Physics 171:822-842. https://doi.org/10.1006/jcph.2001.6813.
8. Le, D. V.; Khoo, B. C.; Peraire, J. 2006. An immersed interface method for viscous incompressible flows involving rigid and flexible boundaries, Journal of Computational Physics 220: 109-138. https://doi.org/10.1016/j.jcp.2006.05.004.

9. Calhoun, D. 2002. Cartesian grid method for solving the two-dimensional streamfunction-vorticity equations in irregular regions, Journal of Computational Physics 176:231-275. https://doi.org/10.1006/jcph.2001.6970.

10. Li, Z.; Wang, A. 2003. A fast finite difference method for solving Navier-Stokes equations on irregular domains, Communications in Mathematical Sciences 1:180-196. https://doi.org/10.1016/j.cms.2003.05.002.

11. Russell, Z. J; Wang, A. 2003. A Cartesian grid method for modeling multiple moving objects in 2D incompressible viscous flow, Journal of Computational Physics 191:177-205. https://doi.org/10.1016/S0021-9991(03)00310-3.

12. Xu, S.; Wang, Z. J. 2008. A 3D immersed interface method for fluid-solid interaction, Transactions of Mechanical Engineering 197: 2068-2086.

https://doi.org/10.1016/j.cma.2007.06.012.

13. Ye, T.; Mittal, R.; Udaykumar, H. S.; Shyy, W. 1999. An accurate Cartesian grid method for viscous incompressible flows with complex immersed boundary, Journal of Computational Physics 156:209-240. https://doi.org/10.1006/jcph.1999.6356.

14. Udaykumar, H. S.; Mittal, R.; Rampunggoon, P.; Khanna, A. 2001. A sharp interface Cartesian grid method for simulating flows with complex moving boundaries, Journal of Computational Physics 174:345380. https://doi.org/10.1006/jcph.2001.6916.

15. Jayathilake, P. G.; Khoo, B. C.; Zhijun, T. 2009.Capsule-substrate adhesion in the presence of osmosis by the immersed interface method, International Conference on Computational Fluid Dynamics19:54-65. https://doi.org/10.5281/zenodo.1082035.

16. Gurugubelli, P. S.; Jaiman, R. K.; Khoo, B. C. 2014. Flexible flapping dynamics of parallel elastic plates in a uniform flow: application to energy harvesting devices, In ASME 2014 33rd International Conference on Ocean, Offshore and Arctic Engineering 21:V002T08A038V002T08A038. https://doi.org/10.1115/OMAE2014-23622.

17. Li, Z.; Jaiman, R. K.; Khoo, B. C. 2016. An immersed interface method for flow past circular cylinder in the vicinity of a plane moving wall, International Journal for Numerical Methods in Fluids 81:611-639. https://doi.org/10.1002/fld.4198.

18. Bagchi, P. 2007.Mesoscale simulation of blood flow in small vessels, Biophysical journal 92: 1858-1877. https://doi.org/10.1529/biophysj.106.095042.

19. Bagchi, P.; Johnson, P. C.; Popel, A. S. 2005. Computational fluid dynamic simulation of aggregation of deformable cells in a shear flow, Journal of biomechanical engineering 127: 1070-1080. https://doi.org/10.1115/1.2112907.

20. Kaoui, B.; Harting, J. 2016. Two-dimensional lattice Boltzmann simulations of vesicles with viscosity contrast, Rheologica Acta 55: 465-475. https://doi.org/10.1007/s00397-015-0867-6. 
21. Ghafouri, A.; Esmaily, R.; Alizadeh, A. 2018. Numerical simulation of tank-treading and tumbling motion of red blood cell in the Poiseuille flow in amMicrochannel with and without obstacle, Iranian Journal of Science and Technology, Transactions of Mechanical Engineering 32:1-12.

https://doi.org/10.1007/s40997-018-0233-2.

22. Falavand, J.; Alizadeh, A.; Ghafouri, A. 2018. Modelling of the dynamics of an immersed body in a microchannel with stenosis using the immersed boundary method, Journal of Computational Applied Mechanics 43:43-52. https://doi.org/10.22059/JCAMECH.2018.243247.193.

23. Brown, D. L.; Cortez, R.; Minion, M. 2001. Accurate projection methods for the incompressible NavierStokes equations, Journal of Computational Physics 168:464-499. https://doi.org/10.1006/jcph.2001.6715.

24. Goldstein, D.; Handler, R. 1993. Modeling a no-slip flow with an external force field, Journal of Computational Physics 105:354-364. https://doi.org/10.1006/jcph.1993.1081.

25. Le Veque, R.; Li, Z. 1997. Immersed interface method for Stokes flow with elastic boundaries or surface tension, SIAM Journal on Scientific Computing 18:709735.

https://doi.org/10.1137/S1064827595282532.

26. Coutanceau, M.; Bouard, R. 1977. Experimental determination of the main features of the viscous flow in the wake of a circular cylinder in uniform translation Steady flow, Journal of Fluid Mechanics 79:231-256. https://doi.org/10.1017/S0022112077000135.

27. Pinelli, A.; Naqavi, I. Z.; Piomelli, U.; Favier, J. 2010. Immersed-boundary methods for general finite-difference and finite-volume Navier-Stokes solvers, Journal of Computational Physics229:9073-9091. https://doi.org/10.1016/j.jcp.2010.08.021.

28. Wang, S.; Zhang, W. 2011. An immersed boundary method based on discrete stream function formulation for two-and three-dimensional incompressible flows, Journal of Computational Physics 230:3479-3499. https://doi.org/10.1016/j.jcp.2011.01.045.

29. Braza,M.; Chassaing, P. 1986. Numerical study and physical analysis of the pressure and velocity fields in the near wake of a circular cylinder, Journaloffluidmechanics 165:79-130. https://doi.org/10.1017/S0022112086003014.

30. Ji, C.; Munjiza, A.; Williams, J. 2012. A novel iterative direct-forcing immersed boundary method and its finite volume applications, Journal of Computational Physics 231:1797-1821.

https://doi.org/10.1016/j.jcp.2011.11.010.

31. Fischer, T.; Schmid-Schonbein, H. 1977.Tank treading motion of red blood cell membranes in viscometric flow, Behavior of intracellular and extracellular markers. Blood Cells 3: 351-365. https://doi.org/10.1017/S0022112082002651.

32. Xiong, W.; Zhang, J. 2010. Shear stress variation induced by red blood cell motion in microvessel, Annals of biomedical engineering 38: 2649-2659. https://doi.org/10.1007/s10439-010-0017-3.

33. Ma, G.; Hua, J.; Li, H. 2009. Numerical modeling of the behavior of an elastic capsule in a microchannel flow: The initial motion, Physical Re.
https://doi.org/10.1103/PhysRevE.79.046710.

A. Abid Mattie, As'ad Alizadeh

USING A NON-CONFORMING MESHES METHOD TO SIMULATE AN INTERACTION BETWEEN INCOMPRESSIBLE FLOW AND RIGID AND ELASTIC BOUNDARIES

S u m m a r y

The interaction between incompressible fluids and elastic and rigid boundaries is seen in many medical, engineering and natural issues. The immersed interface method is used as a non-conforming meshes method to simulate such problems. An important advantage of this method is that there is no compulsion to adapt the fluid grids and the boundary grids. First, the flow around a circular cylinder was simulated. As the Reynolds number rises, the vortex dimensions become larger and, as a result, the separation angle of the flow increases. Also, with the Reynolds number increasing, the drag coefficient decreases and the Strouhal number increases. Also observed that the sick cell makes smaller defor-mation, while the normal cell is more deformed and easier passes the stenosis. This results in reduction of the flow rate in stenosis. This behavior is caused by a type of dis-ease called sickle cell anemia.

Keywords: fluid-structure interaction, immersed interface method, incompressible flow, rigid boundary, elastic boundary.

Received March 16, 2019 Accepted August 26, 2019 